\title{
Situation Perception Mediates the Link Between Narcissism and Relationship Satisfaction: Evidence From a Daily Diary Study in Romantic Couples
}

\author{
Katrin Rentzsch \\ Psychologische Hochschule Berlin \\ Larissa L. Wieczorek \\ University of Hamburg \\ Tanja M. Gerlach
}

University of Goettingen and Leibniz ScienceCampus Primate Cognition

Word Count: 4984

Rentzsch, K., Wieczorek, L. L., Gerlach, T. M. (in press). Situation perception mediates the link between narcissism and relationship satisfaction: Evidence from a daily diary study in romantic couples. Social Psychological and Personality Science.

\section{Author Note}

Katrin Rentzsch, Personality Psychology and Psychological Assessment, Psychologische Hochschule Berlin; Larissa L. Wieczorek, Institute of Psychology, University of Hamburg; Tanja M. Gerlach, Department of Psychology, University of Goettingen and Leibniz ScienceCampus Primate Cognition.

Katrin Rentzsch is a professor of psychology at the Psychologische Hochschule Berlin. Her research focuses on personality and social interaction processes. She is also interested in the dynamic interplay between personality and social emotions, such as envy, and the longitudinal development of domain-specific self-evaluations in adulthood. Larissa L. Wieczorek is a $\mathrm{PhD}$ candidate at the University of Hamburg. In her research, she investigates the interplay of social relationships and personality across life. In addition, she is interested in adolescent development, romantic relationships, and sexuality. Tanja M. Gerlach is a senior 
researcher and teacher at the University of Goettingen. Her research focuses on how people establish and maintain romantic relationships and the role individual differences, such as narcissism and romantic ideals, play therein. In addition, she is passionate about research design for the study of close relationships and their development and endocrinology.

We thank Tessa Kohlberg, Aileen Marske, Susanne Rosenheinrich, and Elisabeth Stenzel for help with data collection.

Correspondence concerning this article should be addressed to Katrin Rentzsch, Personality Psychology and Psychological Assessment, Psychologische Hochschule Berlin, Am Köllnischen Park 2, 10179 Berlin, E-Mail: k.rentzsch@phb.de 


\begin{abstract}
Research has shown that diverging romantic relationship outcomes of grandiose narcissism can be explained by differential associations of agentic and antagonistic aspects of narcissism. In this study, we wanted to further investigate the underlying mechanisms by examining how narcissists perceive daily situations with their partner. In an online diary, 171 couples reported on 1941 daily situations experienced together. Analyses revealed that agentic narcissism was positively and antagonistic narcissism was negatively related to daily relationship satisfaction. These effects were differentially linked through distinct situation perceptions: Agentic narcissism was positively linked with relationship satisfaction through perceiving daily situations as, for example, containing more romance, sexuality and love, while antagonistic narcissism was negatively linked with relationship satisfaction through perceiving, for example, more threat, criticism, and accusation. Results are discussed in light of the NARC model and with respect to person-situation transactions in romantic relationships.
\end{abstract}

Keywords: narcissism, situation perception, romantic relationships, relationship satisfaction, diary study 


\section{Situation Perception Mediates the Link Between Narcissism and Relationship Satisfaction: Evidence From a Daily Diary Study in Romantic Couples}

Grandiose narcissism as a personality trait is characterized by an overly positive view of the self, including feelings of self-importance, social power, and entitlement (Back, 2018; Campbell \& Foster, 2007; Krizan \& Herlache, 2018; Morf \& Rhodewalt, 2001). Previous research has revealed diverging romantic relationship outcomes that are associated with grandiose narcissism. On the one hand, individuals high on narcissism tend to captivate with charm and a neat appearance (Campbell \& Campbell, 2009; Holtzman \& Strube, 2010) making them desirable partners (Dufner et al., 2013; Jauk et al., 2016). On the other hand, narcissists' entitlement (Campbell et al., 2004) or lack of commitment (Campbell \& Foster, 2002) may be detrimental to relationship quality (e.g., Campbell et al., 2002; Peterson \& DeHart, 2014). Accordingly, narcissism is potentially both beneficial and harmful to relationship functioning. In line with this, research on romantic couples has found positive (e.g., Sedikides et al., 2004), negative (e.g., Casale et al., 2019), and null associations (e.g., Campbell \& Foster, 2002) between narcissism and relationship quality.

Recent research has shown that these conflicting results can be explained by differential associations of agentic (e.g., assertiveness, charmingness, entertaining qualities) and antagonistic (e.g., selfishness, low propensity to forgive, insensitivity) aspects of grandiose narcissism (Back et al., 2013; Wurst et al., 2017). Accounting for this heterogeneous nature, the Narcissistic Admiration and Rivalry Concept (NARC; Back et al., 2013) distinguishes two positively correlated dimensions of the construct: narcissistic admiration and rivalry. Both dimensions relate to social strategies serving the goal to maintain a grandiose self yet include distinct affective-motivational, cognitive, and behavioral processes: While narcissistic admiration focuses on gaining social admiration (assertive selfenhancement), narcissistic rivalry focuses on avoiding social failure (antagonistic selfdefense). By disentangling the self-regulatory processes that constitute the agentic and 
antagonistic aspects of grandiose narcissism, the NARC provides a parsimonious explanation for the diversity of narcissistic correlates and consequences.

In this study, we attempt to replicate findings suggesting a positive link of admiration and a negative link of rivalry with relationship satisfaction (Back et al., 2013; Wurst et al., 2017). Moreover, we want to extend these findings by investigating the underlying mechanisms. In particular, to better understand how grandiose narcissism affects relationship satisfaction, we take a closer look at the interplay between narcissistic personality and situations. We focus on the cognitive processes involved in narcissistic admiration and rivalry and examine how narcissists perceive daily situations with their partner.

People individually process situational cues and form their own psychological representation of a given situation (Rauthmann, Sherman, \& Funder, 2015). As Furr and Funder (in press) note, a person's perception of the situation might have a more direct effect on their reaction than the situation itself. Situations differ regarding actual and perceived affordances (Reis \& Holmes, 2019), i.e. objective cues or subjectively relevant characteristics that may afford certain reactions from persons (Blum et al., 2018; Rauthmann, Sherman, \& Funder, 2015). In recent years, numerous models of situation perception, capturing these different characteristics, have been developed (e.g., Gerpott et al., 2018; Parrigon et al., 2017; Rauthmann et al., 2014). Among them is the DIAMONDS taxonomy, which describes the eight dimensions Duty (something needs to be done), Intellect (intellectual capacity can be demonstrated), Adversity (someone is blamed or threatened), roMance ${ }^{1}$ (opportunity for romantic attraction is given), pOsitivity (positive feelings are likely), Negativity (negative feelings are likely), Deception (mistrust and deception arise) and Sociality (social interaction is involved; Rauthmann et al., 2014). Research suggests that situation perception affects relationship outcomes: Using hypothetical scenarios involving the participants' romantic partner, Finn et al. (2013) found that more negative interpretations of imagined situations related to reduced relationship satisfaction. Thus, cognitive processes including the individual 
perception of situations likely have an impact on relationship satisfaction in real-life scenarios, too.

In terms of personality-situation transactions, it has been shown that personality shapes the way situations are experienced (Furr \& Funder, in press; Hong et al., 2020; Sherman et al., 2013). Regarding narcissism, Sherman and colleagues (2013) found participants scoring higher on the Narcissistic Personality Inventory (NPI) to perceive situations as opportunities to be the center of attention, to demonstrate intellect, to express their charm, as requiring assertiveness and being sexually meaningful. However, the NPI mainly captures agentic aspects of narcissism (Back et al., 2013) and it remains unclear which situation construal is specific to its antagonistic component, rivalry. Since personality relates to situation perception (Hong et al., 2020; Sherman et al., 2013) and this kind of cognitive processing may affect relationship experiences (Finn et al., 2013), we expected that narcissism links to relationship satisfaction through a specific construal of situations experienced with the partner. More specifically and taking the different aspects of grandiose narcissism into account, we expected that the differential associations of agentic and antagonistic aspects of narcissism and relationship satisfaction may be mediated through a distinct set of situation perceptions.

The self-regulatory processes outlined by the NARC (Back et al., 2013) include cognitions such as grandiose fantasies as an aspect of narcissistic admiration and devaluation of others as an aspect of rivalry. In line with this, Wurst et al. (2017) found that participants higher on admiration not only enhanced themselves, but also evaluated their partners in a more positive manner. At the same time, those higher on rivalry evaluated their partners less favorably. Starting with the broader classification of valence, we predicted that admiration positively relates to relationship satisfaction through a more positive (Hypothesis 1 ) and a less negative construal of the situation (Hypothesis 2). Vice versa we expected that rivalry negatively links to relationship satisfaction through a less positive (Hypothesis 3 ) and a more 
negative situational construal (Hypothesis 4). Regarding admiration, we further anticipated a positive association between admiration and relationship satisfaction via a more romantic view on situations (Hypothesis 5). We based our expectation on research employing the NPI, a narcissism measure heavy on agentic content (Back et al., 2013; Wright \& Edershile, 2018), which has found that people with more narcissistic dispositions likely perceive situations as opportunities for flirting and sexual approaches (Sherman et al., 2013). For rivalry, we predicted two distinct associations. First, rivalry has been proposed to go along with a tendency to readily perceive ego-threat and individuals scoring high on rivalry have been found to perceive others as more aggressive (Back et al., 2013) and less respectful (Vrabel et al., 2019). Therefore, we expected rivalry to be negatively linked to relationship satisfaction through a tendency to construe situations as adverse or threatening (Hypothesis 6). Second, the so-called Dark Triad of personality (i.e. sub-clinical narcissism, machiavellianism, and psychopathy) has been linked to situation perceptions that contain mistrust, deception, and lying (Rauthmann et al., 2014). Correspondingly, Back et al. (2013) have found those high on rivalry scoring higher on interpersonal distrust and perceiving others as less trustworthy. Accordingly, we expected a negative link between rivalry and relationship satisfaction through experiencing more deception (Hypothesis 7). For the situational dimensions Duty, Intellect, and Sociality, no specific relationships with either admiration or rivalry were expected. Nonetheless, we decided to analyze whether the influence of both facets of grandiose narcissism is mediated through any of the eight dimensions of the DIAMONDS model. None of our hypotheses were preregistered.

\section{Methods}

We used the open source survey framework formr to implement our diary study (https://formr.org; Arslan et al., 2019). Informed consent and the dependency of data due to the dyadic design only allow limited data sharing for scientific purposes. Data can be requested from the first author. 


\section{Participants}

Participants were recruited via announcements in university newsletters, social media, and mailing lists. The study was advertised as a study about "personality and situation perception in romantic relationships." Participants were informed that the study consisted of an online pre-questionnaire and a 14 days online diary, which would take about five to ten minutes per day and per partner to complete. As an incentive, participants received partial course credit (where applicable) and feedback on their personality once they had completed the study. In addition, couples of which both partners completed the study had the chance to win a $300 €$ event voucher. Based on practical constraints, we initially sought to recruit a minimum of 100 couples. $^{2}$

A total of 203 couples ( $N=406$ participants) completed the intake session. In the diary, $N=2276$ situations were reported by 180 couples (i.e. $N=4552$ individual situation descriptions stemming from 360 participants). An independent coder rated each situation description with respect to whether both dyad members' descriptions referred to the same situation. As a result, 254 situations had to be excluded from analyses because of non-matching situation descriptions. To enable analyses for distinguishable dyads (see Bolger \& Laurenceau, 2013), only heterosexual couples were included, resulting in 1941 matching situation descriptions from 171 heterosexual couples. $^{3}$ Average age of participants was 28.9 years $(S D=11.9$, Min=17, $\mathrm{Max}=74 ; M_{\mathrm{age}}=29.9$ years for male participants and 27.9 years for female participants). Seventy participants reported to be married, 263 to be in a relationship, and seven participants to be in a relationship classified as open. One hundred seventy-three $(51 \%)$ participants reported living together, whereas 169 (49\%) reported not living together. On average, participants had been in their relationship for 5.3 years $(S D=7.2, \mathrm{Min}=1, \mathrm{Max}=49)$.

\section{Procedure and Measures}

The study consisted of an intake session and 14 consecutive days. On each day, partners filled out the questionnaires individually. At intake, a number of demographic items 
and personality measures including grandiose narcissism were assessed. Assertive and antagonistic narcissism were assessed with the Narcissistic Admiration and Rivalry Questionnaire (NARQ; Back et al., 2013). Responses were given on 6-point Likert-type scales ranging from 1 (not agree at all) to 6 (agree completely).

On each diary day, participants were asked to report on a situation they had experienced with their partner at $7 \mathrm{pm}$ on the given day (see Guillaume et al., 2016). If participants did not spend that time with their partner, they were instructed to choose another recent situation they had spent together before or after. The importance of both partners describing the same situation was emphasized and the couple was allowed to communicate in order to agree upon one situation. In the diary, participants were instructed to describe the situation briefly in at least two to three sentences, to specify the date of the situation and to specify the context in which the situation was experienced (e.g., in person or via phone). Following the situation description, participants were asked to complete the 8-item $S 8$-II (Rauthmann \& Sherman, 2016) to describe their individual perceptions of the actual situation. They were instructed not to talk with their partners about the items. The S8-II assesses eight dimensions of a given situation (“The Situation contains...”): "Work, tasks, duties”(Duty), "Intellectual, aesthetic, profound things"(Intellect), "Threat, criticism, accusation"(Adversity), "Romance, sexuality, love"(roMance), "Positive, pleasant, nice things"(pOsitivity), "Negative things, unpleasant things, bad feelings”(Negativity), "Deceit, lie, dishonesty”(Deception), "Communication, interaction, social relationships"(Sociality). Responses were made on a Likert-type scale from 1 (not at all) to 7 (totally), indicating how much every item applied to the situation described (Rauthmann \& Sherman, 2016).

To assess daily relationship satisfaction, participants completed three items of the relationship assessment scale (RAS; Hendrick, 1981; German adaptation by Sander \& Böcker, 1993), all of which referred to their relationship satisfaction on the specific day. All 
items were scored on a five-point Likert scale from 1 (low relationship satisfaction) to 5 (high relationship satisfaction).

The final questionnaire at day 14 was identical to the daily questionnaires, except that at the end, participants additionally completed personality measures and a trait measure of relationship satisfaction, all of which are not relevant for the present article.

\section{Analytic Strategy}

To account for the dyadic data structure, we used multilevel models for dyadic diary data from distinguishable dyads (Laurenceau \& Bolger, 2012). These models take dependencies of errors between dyad members at each point of assessment into account. All multilevel analyses were carried out in Mplus Version 8 (L. Muthén \& Muthén, 1998-2017). In our models, we specified Bayesian estimation (B. Muthén, 2010). For all parameters, we chose non-informative priors. Further, we calculated the median of the posterior distribution (expected a posteriori) as the point estimate and the $95 \%$ Bayesian credibility interval (CI) as the interval estimate for all parameters. We also provide a one-tailed p-value based on the posterior distribution. We report effects as statistically significant if their one-tailed p-value is below .025 .

The multilevel structure of the dyadic diary data included daily situations ( $N=1941$, Level 1) nested in dyads ( $N=171$, Level 2$)$. Since the multilevel model for dyadic diary data (Laurenceau \& Bolger, 2012) is an adaptation of the actor-partner interdependence model (Kenny et al., 2006), daily reports such as the outcome variable daily relationship satisfaction were modelled for every partner separately, and simultaneously. Descriptive statistics and intercorrelations are displayed in Table 1. 


\section{Table 1}

Descriptive Statistics and Intercorrelations of Study Variables Measured in Men and Women

\begin{tabular}{|c|c|c|c|c|c|c|c|c|c|c|c|c|c|c|c|c|c|c|c|c|c|c|c|c|}
\hline & & $M$ & $S D$ & 1 & 2 & 3 & 4 & 5 & 6 & 7 & 8 & 9 & 10 & 11 & 12 & 13 & 14 & 15 & 16 & 17 & 18 & 19 & 20 & 21 \\
\hline 1 & $\operatorname{Riv}_{m}$ & 2.29 & 0.78 & - & & & & & & & & & & & & & & & & & & & & \\
\hline 2 & $\operatorname{Riv}_{f}$ & 1.94 & 0.62 & .09 & - & & & & & & & & & & & & & & & & & & & \\
\hline 3 & $\mathrm{Adm}_{\mathrm{m}}$ & 3.13 & 0.80 & .33 & .00 & - & & & & & & & & & & & & & & & & & & \\
\hline 4 & $\operatorname{Adm}_{\mathrm{f}}$ & 2.84 & 0.78 & .09 & .22 & .22 & - & & & & & & & & & & & & & & & & & \\
\hline 5 & Dutym & 3.45 & 2.05 & .19 & -.07 & .07 & -.07 & - & & & & & & & & & & & & & & & & \\
\hline 6 & Dutyf $_{f}$ & 3.30 & 2.13 & -.03 & .09 & -.19 & -.04 & .40 & - & & & & & & & & & & & & & & & \\
\hline 7 & Intellect $_{m}$ & 3.33 & 1.79 & .05 & -.06 & .17 & .17 & .40 & .17 & - & & & & & & & & & & & & & & \\
\hline 8 & Intellectf & 3.06 & 1.83 & -.15 & -.05 & .02 & .24 & -.05 & .43 & .46 & - & & & & & & & & & & & & & \\
\hline 9 & Adversitym & 1.86 & 1.47 & .22 & -.08 & -.06 & -.07 & .49 & .20 & .20 & .10 & - & & & & & & & & & & & & \\
\hline 10 & Adversityf & 1.80 & 1.47 & .03 & .07 & -.10 & -.01 & .10 & .37 & .02 & .38 & .37 & - & & & & & & & & & & & \\
\hline 11 & RoMance $_{\mathrm{m}}$ & 3.43 & 1.91 & .06 & .00 & .21 & .19 & .03 & -.18 & .48 & .25 & .04 & -.03 & - & & & & & & & & & & \\
\hline 12 & RoMance $_{\mathrm{f}}$ & 3.49 & 1.89 & .02 & .02 & .15 & .24 & -.16 & -.12 & .31 & .48 & -.04 & .04 & .64 & - & & & & & & & & & \\
\hline 13 & POsitivitym & 5.08 & 1.61 & -.15 & -.04 & .16 & .16 & -.02 & -.21 & .37 & .18 & -.34 & -.43 & .55 & .53 & - & & & & & & & & \\
\hline 14 & POsitivityf & 5.04 & 1.73 & -.16 & .02 & .06 & .19 & -.27 & -.07 & .24 & .33 & -.34 & -.34 & .36 & .61 & .63 & - & & & & & & & \\
\hline 15 & Negativitym & 2.46 & 1.70 & .26 & -.06 & -.11 & -.15 & .56 & .23 & .17 & .05 & .78 & .32 & .08 & -.12 & -.34 & -.34 & - & & & & & & \\
\hline 16 & Negativityf & 2.47 & 1.78 & .09 & .13 & -.08 & .00 & .18 & .42 & .06 & .27 & .41 & .79 & .05 & -.03 & -.40 & -.41 & .50 & - & & & & & \\
\hline 17 & Deception $_{m}$ & 1.28 & 0.86 & .32 & -.11 & .07 & -.02 & .40 & .25 & .29 & .13 & .75 & .19 & .19 & .10 & -.14 & -.09 & .71 & .28 & - & & & & \\
\hline 18 & Deceptionf $_{\mathrm{f}}$ & 1.23 & 0.85 & .14 & -.03 & .05 & .13 & .09 & .08 & -.09 & .15 & .22 & .52 & -.14 & .04 & -.28 & -.29 & .23 & .57 & .14 & - & & & \\
\hline 19 & Socialitym & 5.50 & 1.42 & -.14 & -.05 & .04 & -.10 & .11 & -.03 & .33 & .20 & .00 & -.15 & .23 & .19 & .42 & .23 & .08 & -.14 & -.02 & -.17 & - & & \\
\hline 20 & Socialityf & 5.66 & 1.44 & -.20 & -.09 & -.06 & -.02 & .01 & .23 & .25 & .50 & -.03 & .02 & .19 & .44 & .27 & .54 & -.02 & -.06 & -.03 & -.24 & .41 & - & \\
\hline 21 & Rel.Sat ${ }_{\mathrm{m}}$ & 4.00 & 0.84 & -.17 & -.05 & .07 & .04 & -.02 & -.08 & .25 & .17 & -.40 & -.20 & .37 & .33 & .80 & .43 & -.27 & -.22 & -.23 & -.12 & .35 & .24 & - \\
\hline 22 & Rel.Sat $_{f}$ & 3.99 & 0.90 & -.15 & .00 & -.06 & .15 & -.09 & -.05 & .29 & .24 & -.31 & -.17 & .36 & .47 & .61 & .60 & -.25 & -.29 & -.03 & -.30 & .31 & .50 & .62 \\
\hline
\end{tabular}

Note. Intercorrelations refer to the maximum-likelihood estimated between zero-order correlations, respectively. Rel.Sat $=$ relationship satisfaction, Adm $=$ narcissistic admiration, Riv $=$ narcissistic rivalry, $\mathrm{m}=$ male, $\mathrm{f}=$ female, $N_{\text {within }}=1941, N_{\text {between }}=171$. 
We first examined the unique associations of narcissistic admiration and rivalry with both partners' relationship satisfaction . Admiration and rivalry were grand-mean centered prior to analyses. Since we were interested in the link between participants' narcissism and relationship satisfaction, we focused on actor-effects in particular. As some studies on narcissism in couples found actor- as well as partner-effects (Casale et al., 2019; GerwitzMeydan \& Finzi-Dottan, 2018), we also report partner-effects in the following (Kenny et al., 2006).

In the multiple mediation model, all dimensions of situation perceptions were included simultaneously. Analyses were based on 2-1-1 mediation with random intercepts (Preacher et al., 2010). If a 1-1 path showed a significant difference regarding its within- and betweencomponent, the between-component of the 1-1 path was included in computing the indirect effect. Paths which did not differ regarding their within- and between-components were treated accordingly in the computation of the indirect effect. All actor- and partner-effects were constrained to be equal across men and women. To investigate gender-specific effects, we ran additional models with no constraints, all of which are reported in the Supplemental Material S1 (https://osf.io/pn58t/?view_only=daa4ac1d6b8f42789f37e9ac6d39cfd6) in more detail. In the following, unstandardized estimates are reported. Standardized estimates can be obtained from the Supplemental Material S1.

Inspired by reviewer suggestions, we ran exploratory supplemental analyses, all of which are presented in Supplement S2 in more detail (https://osf.io/pn58t/?view_only=daa4ac1d6b8f42789f37e9ac6d39cfd6). Here, we focused on the hypothesized actor-effects, and included the modelling of age, living together and relationship length as covariates, and moderation analyses with relationship length as moderator. We also investigated whether the hypothesized mediation effects were uniquely due to the specific content of every DIAMONDS dimension or due to broader components of positive and negative situation perception. Furthermore, we ran additional analyses focusing 
on the subjective construal vs. objective characteristics of a social situation as mediators. We also report models including every DIAMONDS dimension as a single mediator separately. An overview of all measures, analyses, code and output is provided on the OSF (https://osf.io/pn58t/?view_only=daa4ac1d6b8f42789f37e9ac6d39cfd6).

\section{Results}

Intraclass correlations from a null model revealed that $39 \%$ of the variance in male spouses' daily relationship satisfaction and $28 \%$ in female spouses' daily relationship satisfaction was due to differences between dyads. Thus, relationship satisfaction was more variable across situations than between dyads.

\section{Narcissism and Relationship Satisfaction}

Results revealed that narcissistic admiration was positively related to relationship satisfaction and narcissistic rivalry was negatively related to relationship satisfaction (Table 2). In particular, individuals who scored higher on admiration reported higher relationship satisfaction than those lower on admiration $(\mathrm{b}=0.099, p=.020,95 \% \mathrm{CI}[0.016,0.183])$. Spouses who scored higher on rivalry reported lower relationship satisfaction than those scoring lower on rivalry $(\mathrm{b}=-0.092, p=.010,95 \% \mathrm{CI}[-0.182,-0.004])$. Our results, thus, replicate findings from previous research (Back et al., 2013; Wurst et al., 2017). Furthermore, results from analyses without constraints of gender-effects showed that the negative effect of rivalry was significant in male spouses only $(b=-0.125, p=.020,95 \% \mathrm{CI}[-0.265,-0.002]$; see Table S1c of the Supplement S1 for more detail). The different facets of narcissism in one partner, however, were not significantly related to the relationship satisfaction of the other partner (partner-effects, $p \mathrm{~s} \geq .80$ ). 


\section{Table 2}

Narcissistic Admiration and Rivalry Predict Relationship Satisfaction

\begin{tabular}{|c|c|c|c|c|c|}
\hline & Estimate & $\begin{array}{c}\text { Posterior } \\
\text { SD }\end{array}$ & $\begin{array}{c}\text { One-tailed } \\
\text { p-value }\end{array}$ & $\mathrm{LCL}$ & UCL \\
\hline \multicolumn{6}{|c|}{ Rel.Sat (male) } \\
\hline $\mathrm{Adm}_{\mathrm{m}}$ & 0.099 & 0.045 & .020 & 0.016 & 0.183 \\
\hline $\operatorname{Adm}_{f}$ & -0.022 & 0.039 & .270 & -0.107 & 0.050 \\
\hline $\operatorname{Riv}_{m}$ & -0.092 & 0.042 & .010 & -0.182 & -0.004 \\
\hline $\operatorname{Riv}_{f}$ & -0.056 & 0.040 & .080 & -0.138 & 0.016 \\
\hline \multicolumn{6}{|c|}{ Rel.Sat (female) } \\
\hline $\operatorname{Adm}_{\mathrm{f}}$ & 0.099 & 0.045 & .020 & 0.016 & 0.183 \\
\hline $\mathrm{Adm}_{\mathrm{m}}$ & -0.022 & 0.039 & .270 & -0.107 & 0.050 \\
\hline $\operatorname{Riv}_{f}$ & -0.092 & 0.042 & .010 & -0.182 & -0.004 \\
\hline $\operatorname{Riv}_{m}$ & -0.056 & 0.040 & .080 & -0.138 & 0.016 \\
\hline
\end{tabular}

Note. Cells present unstandardized estimates from multilevel models for dyadic diary data for distinguishable dyads including constrained actor- and partner-effects across gender. Significant effects are highlighted in bold. ${ }^{5}$ Effects of predictors referring to the same gender as the outcome represent actor-effects. Effects of predictors referring to a different gender as the outcome represent partner-effects. Rel.Sat = relationship satisfaction, Adm $=$ narcissistic admiration, $\mathrm{Riv}=$ narcissistic rivalry, $\mathrm{m}=$ male, $\mathrm{f}=$ female, $\mathrm{LCL}=$ lower confidence limit $(95 \%$ CI), UCL $=$ upper confidence limit $(95 \% \mathrm{CI}) . N_{\text {within }}=1941, N_{\text {between }}=171$.

\section{Narcissism, Situation Perception and Relationship Satisfaction}

Our analyses revealed that the different facets of narcissism were differentially linked to relationship satisfaction through a distinct set of situation perceptions. Results relating to the different mediation paths (i.e. effects of situation perception on relationship satisfaction and effects of admiration and rivalry on situation perception) can be obtained from Table 3 . The mediated effects (i.e. the indirect effects for admiration and rivalry through situation perception) are summarized in Table 4 and further discussed in the following. 
Table 3

Estimates From Multiple Mediator Model Linking Narcissistic Admiration and Rivalry to Relationship Satisfaction Through Situation Perception

\begin{tabular}{lccccc}
\hline Estimate & $\begin{array}{c}\text { Posterior } \\
\text { SD }\end{array}$ & $\begin{array}{c}\text { One-tailed } \\
\text { p-value }\end{array}$ & LCL & UCL \\
\hline Within Level & & &
\end{tabular}

Situation perception predicts relationship satisfaction

\begin{tabular}{|c|c|c|c|c|c|}
\hline \multicolumn{6}{|l|}{ Rel.Sat (male) } \\
\hline Dutym $_{m}$ & 0.023 & 0.006 & $<.001$ & 0.010 & 0.035 \\
\hline Dutyf $_{f}$ & 0.006 & 0.006 & .175 & -0.006 & 0.016 \\
\hline Intellect $_{\mathrm{m}}$ & 0.031 & 0.006 & $<.001$ & 0.018 & 0.041 \\
\hline Intellect $_{\mathrm{f}}$ & 0.004 & 0.007 & .315 & -0.011 & 0.016 \\
\hline Adversity $_{m}$ & -0.080 & 0.009 & $<.001$ & -0.098 & -0.063 \\
\hline Adversity $_{f}$ & -0.049 & 0.009 & $<.001$ & -0.067 & -0.033 \\
\hline RoMance $_{\mathrm{m}}$ & 0.097 & 0.007 & $<.001$ & 0.081 & 0.109 \\
\hline RoMance $_{f}$ & 0.016 & 0.007 & .010 & 0.002 & 0.029 \\
\hline POsitivitym $_{m}$ & 0.087 & 0.009 & $<.001$ & 0.068 & 0.106 \\
\hline POsitivityf & 0.002 & 0.009 & .435 & -0.015 & 0.020 \\
\hline Negativitym $_{m}$ & -0.048 & 0.008 & $<.001$ & -0.067 & -0.032 \\
\hline Negativityf $_{f}$ & 0.006 & 0.009 & .250 & -0.016 & 0.022 \\
\hline Deception $_{\mathrm{m}}$ & -0.004 & 0.013 & .340 & -0.033 & 0.017 \\
\hline Deception $_{\mathrm{f}}$ & 0.038 & 0.013 & $<.001$ & 0.014 & 0.061 \\
\hline Socialitym $_{\mathrm{m}}$ & 0.072 & 0.009 & $<.001$ & 0.053 & 0.088 \\
\hline Socialityf & 0.018 & 0.008 & $<.001$ & 0.003 & 0.033 \\
\hline \multicolumn{6}{|l|}{ Rel.Sat (female) } \\
\hline Duty $_{m}$ & 0.006 & 0.006 & .175 & -0.006 & 0.016 \\
\hline Duty $_{f}$ & 0.023 & 0.006 & $<.001$ & 0.010 & 0.035 \\
\hline Intellect $_{\mathrm{m}}$ & 0.004 & 0.007 & .315 & -0.011 & 0.016 \\
\hline Intellect $_{f}$ & 0.031 & 0.006 & $<.001$ & 0.018 & 0.041 \\
\hline Adversitym $_{m}$ & -0.049 & 0.009 & $<.001$ & -0.067 & -0.033 \\
\hline Adversityf $_{f}$ & -0.080 & 0.009 & $<.001$ & -0.098 & -0.063 \\
\hline RoMance $_{\mathrm{m}}$ & 0.016 & 0.007 & .010 & 0.002 & 0.029 \\
\hline RoMancef $_{\mathrm{f}}$ & 0.097 & 0.007 & $<.001$ & 0.081 & 0.109 \\
\hline POsitivitym & 0.002 & 0.009 & .435 & -0.015 & 0.020 \\
\hline POsitivityf & 0.087 & 0.009 & $<.001$ & 0.068 & 0.106 \\
\hline Negativitym $_{m}$ & 0.006 & 0.009 & .250 & -0.016 & 0.022 \\
\hline Negativity $_{f}$ & -0.048 & 0.008 & $<.001$ & -0.067 & -0.032 \\
\hline Deception $_{\mathrm{m}}$ & 0.038 & 0.013 & $<.001$ & 0.014 & 0.061 \\
\hline Deception $_{\mathrm{f}}$ & -0.004 & 0.013 & .340 & -0.033 & 0.017 \\
\hline Socialitym $_{\mathrm{m}}$ & 0.018 & 0.008 & $<.001$ & 0.003 & 0.033 \\
\hline Socialityf $_{f}$ & 0.072 & 0.009 & $<.001$ & 0.053 & 0.088 \\
\hline
\end{tabular}




\section{$\underline{\text { Between Level }}$}

Situation perception, narcissistic admiration and rivalry predict relationship satisfaction

\begin{tabular}{|c|c|c|c|c|c|}
\hline \multicolumn{6}{|l|}{ Rel.Sat (male) } \\
\hline $\operatorname{Riv}_{m}$ & -0.038 & 0.042 & .140 & -0.123 & 0.049 \\
\hline $\operatorname{Riv}_{f}$ & -0.003 & 0.053 & .485 & -0.125 & 0.095 \\
\hline $\mathrm{Adm}_{\mathrm{m}}$ & -0.020 & 0.045 & .360 & -0.109 & 0.062 \\
\hline $\operatorname{Adm}_{f}$ & -0.053 & 0.045 & .145 & -0.142 & 0.041 \\
\hline Dutym $_{m}$ & 0.023 & 0.006 & $<.001$ & 0.010 & 0.035 \\
\hline Dutyf $_{f}$ & 0.006 & 0.006 & .175 & -0.006 & 0.016 \\
\hline Intellect $_{\mathrm{m}}$ & 0.031 & 0.006 & $<.001$ & 0.018 & 0.041 \\
\hline Intellect $_{f}$ & 0.004 & 0.007 & .315 & -0.011 & 0.016 \\
\hline Adversitym & -0.158 & 0.065 & .020 & -0.268 & -0.015 \\
\hline Adversityf $_{f}$ & -0.049 & 0.009 & $<.001$ & -0.067 & -0.033 \\
\hline RoMance $_{\mathrm{m}}$ & 0.097 & 0.007 & $<.001$ & 0.081 & 0.109 \\
\hline RoMance $_{f}$ & 0.016 & 0.007 & .010 & 0.002 & 0.029 \\
\hline POsitivitym & 0.320 & 0.047 & $<.001$ & 0.231 & 0.406 \\
\hline POsitivity $_{f}$ & 0.002 & 0.009 & .435 & -0.015 & 0.020 \\
\hline Negativitym $_{m}$ & -0.008 & 0.059 & .440 & -0.123 & 0.125 \\
\hline Negativity $_{f}$ & 0.006 & 0.009 & .250 & -0.016 & 0.022 \\
\hline Deception $_{\mathrm{m}}$ & -0.004 & 0.013 & .340 & -0.033 & 0.017 \\
\hline Deception $_{\mathrm{f}}$ & 0.038 & 0.013 & $<.001$ & 0.014 & 0.061 \\
\hline Socialitym & 0.072 & 0.009 & $<.001$ & 0.053 & 0.088 \\
\hline Socialityf $_{f}$ & 0.018 & 0.008 & $<.001$ & 0.003 & 0.033 \\
\hline \multicolumn{6}{|l|}{ Rel.Sat (female) } \\
\hline $\operatorname{Riv}_{m}$ & 0.000 & 0.046 & .500 & -0.086 & 0.095 \\
\hline $\operatorname{Riv}_{f}$ & 0.024 & 0.056 & .380 & -0.103 & 0.125 \\
\hline $\mathrm{Adm}_{\mathrm{m}}$ & -0.082 & 0.048 & .050 & -0.182 & 0.011 \\
\hline $\operatorname{Adm}_{\mathrm{f}}$ & 0.019 & 0.048 & .385 & -0.084 & 0.113 \\
\hline Dutym $_{m}$ & 0.006 & 0.006 & .175 & -0.006 & 0.016 \\
\hline Dutyf $_{f}$ & 0.023 & 0.006 & $<.001$ & 0.010 & 0.035 \\
\hline Intellect $_{\mathrm{m}}$ & 0.004 & 0.007 & .315 & -0.011 & 0.016 \\
\hline Intellect $_{f}$ & 0.031 & 0.006 & $<.001$ & 0.018 & 0.041 \\
\hline Adversitym $_{m}$ & -0.049 & 0.009 & $<.001$ & -0.067 & -0.033 \\
\hline Adversity $_{f}$ & -0.158 & 0.065 & .020 & -0.268 & -0.015 \\
\hline RoMance $_{\mathrm{m}}$ & 0.016 & 0.007 & .010 & 0.002 & 0.029 \\
\hline RoMance $_{f}$ & 0.097 & 0.007 & $<.001$ & 0.081 & 0.109 \\
\hline POsitivitym & 0.002 & 0.009 & .435 & -0.015 & 0.020 \\
\hline POsitivityf & 0.320 & 0.047 & $<.001$ & 0.231 & 0.406 \\
\hline Negativitym $_{m}$ & 0.006 & 0.009 & .250 & -0.016 & 0.022 \\
\hline Negativityf $_{f}$ & -0.008 & 0.059 & .440 & -0.123 & 0.125 \\
\hline Deception $_{\mathrm{m}}$ & 0.038 & 0.013 & $<.001$ & 0.014 & 0.061 \\
\hline Deception $_{f}$ & -0.004 & 0.013 & .340 & -0.033 & 0.017 \\
\hline Socialitym $_{\mathrm{m}}$ & 0.018 & 0.008 & $<.001$ & 0.003 & 0.033 \\
\hline Socialityf & 0.072 & 0.009 & $<.001$ & 0.053 & 0.088 \\
\hline
\end{tabular}


Narcissistic admiration and rivalry predict situation perception

Duty (male)

$\begin{array}{lrrrrr}\operatorname{Adm}_{\mathrm{m}} & 0.005 & 0.081 & .450 & -0.143 & 0.170 \\ \operatorname{Adm}_{\mathrm{f}} & -0.145 & 0.082 & .035 & -0.310 & 0.025 \\ \operatorname{Riv}_{\mathrm{m}} & \mathbf{0 . 1 9 4} & 0.089 & .015 & 0.016 & 0.339 \\ \operatorname{Riv}_{\mathrm{f}} & -0.023 & 0.086 & .415 & -0.193 & 0.128\end{array}$

Intellect (male)

$\begin{array}{lrrrrr}\operatorname{Adm}_{\mathrm{m}} & \mathbf{0 . 2 5 6} & 0.071 & <.001 & 0.122 & 0.395 \\ \operatorname{Adm}_{\mathrm{f}} & 0.129 & 0.084 & .095 & -0.074 & 0.262 \\ \operatorname{Riv}_{\mathrm{m}} & -0.058 & 0.085 & .220 & -0.229 & 0.100 \\ \operatorname{Riv}_{\mathrm{f}} & \mathbf{- 0 . 2 0 2} & 0.082 & .015 & -0.348 & -0.023\end{array}$

Adversity (male)

$\mathrm{Adm}_{\mathrm{m}}$

$\operatorname{Adm}_{\mathrm{f}}$

$\operatorname{Riv}_{m}$

$\operatorname{Riv}_{f}$

$-0.049 \quad 0.056$

$-0.062 \quad 0.053$

$\mathbf{0 . 1 6 2} 0.053$

$-0.007 \quad 0.055$

.165

.130

$<.001$

.475

$-0.147$

$-0.163$

0.058

$-0.113$

0.061

0.047

0.268

0.117

RoMance (male)

$\operatorname{Adm}_{m}$
$\operatorname{Adm}_{\mathrm{f}}$
$\operatorname{Riv}_{\mathrm{m}}$
$\operatorname{Riv}_{\mathrm{f}}$

POsitivity (male)

$\operatorname{Adm}_{m}$
$\operatorname{Adm}_{f}$
$\operatorname{Riv}_{m}$
$\operatorname{Riv}_{f}$

Negativity (male)

$\mathrm{Adm}_{\mathrm{m}}$

$\operatorname{Adm}_{\mathrm{f}}$

$\operatorname{Riv}_{m}$

Rivf

$\begin{array}{rr}\mathbf{0 . 2 4 6} & 0.074 \\ \mathbf{0 . 1 6 7} & 0.082 \\ -0.027 & 0.095 \\ -0.049 & 0.090\end{array}$

$<.001$

.015

.390

.275

0.109

0.404

0.013

0.320

$-0.215$

0.167

$-0.246$

0.126

$\mathbf{0 . 1 6 6} 0.057$

$0.105 \quad 0.060$

$-0.117 \quad 0.068$

$\mathbf{- 0 . 1 3 3} 0.064$

$<.001$

.050

.040

.025

0.056

$-0.011$

0.264

$-0.257$

0.210

$-0.278$

0.018

$-0.001$

Deception (male)

$\begin{array}{lrrrrr}\operatorname{Adm}_{m} & 0.023 & 0.031 & .215 & -0.038 & 0.091 \\ \operatorname{Adm}_{f} & -0.008 & 0.033 & .405 & -0.075 & 0.053 \\ \operatorname{Riv}_{m} & \mathbf{0 . 0 9 6} & 0.035 & <.001 & 0.020 & 0.160 \\ \operatorname{Riv}_{f} & 0.011 & 0.037 & .415 & -0.077 & 0.076\end{array}$

Sociality (male)

$\mathrm{Adm}_{\mathrm{m}}$

$\begin{array}{rr}-0.102 & 0.063 \\ \mathbf{- 0 . 1 3 5} & 0.065 \\ \mathbf{0 . 2 5 0} & 0.066 \\ 0.052 & 0.074\end{array}$

.045

$-0.222$

0.005

.015

$-0.277$

$-0.016$

$<.001$

.220

0.106

0.370

$-0.096$

0.200

$0.050 \quad 0.063$

.190

$-0.093$

0.159 


$\begin{array}{lrlllr}\text { Adm }_{f} & -0.063 & 0.061 & .160 & -0.173 & 0.059 \\ \operatorname{Riv}_{m} & \mathbf{- 0 . 1 3 2} & 0.067 & .015 & -0.263 & -0.015 \\ \operatorname{Riv}_{f} & -0.108 & 0.072 & .040 & -0.268 & 0.010\end{array}$

Duty (female)

$\operatorname{Adm}_{\mathrm{m}}$

$\operatorname{Adm}_{\mathrm{f}}$

Rivm $_{m}$

$\operatorname{Riv}_{f}$

Intellect (female)

$\operatorname{Adm}_{m}$

$\operatorname{Adm}_{\mathrm{f}}$

Riv $_{m}$

$\operatorname{Riv}_{f}$

Adversity (female)

$$
\mathrm{Adm}_{\mathrm{m}}
$$

$\operatorname{Adm}_{\mathrm{f}}$

$\operatorname{Riv}_{m}$

$\operatorname{Riv}_{\mathrm{f}}$

RoMance (female)

$\mathrm{Adm}_{\mathrm{m}}$

$\operatorname{Adm}_{\mathrm{f}}$

$\operatorname{Riv}_{m}$

$\operatorname{Riv}_{f}$

POsitivity (female)

$\operatorname{Adm}_{m}$
$\operatorname{Adm}_{f}$
$\operatorname{Riv}_{m}$
$\operatorname{Riv}_{f}$

Negativity (female)

$\operatorname{Adm}_{\mathrm{m}}$
$\operatorname{Adm}_{\mathrm{f}}$
$\operatorname{Riv}_{\mathrm{m}}$
$\operatorname{Riv}_{\mathrm{f}}$

Adm $_{\mathrm{m}}$
$\operatorname{Adm}_{\mathrm{f}}$
$\operatorname{Riv}_{\mathrm{m}}$
$\operatorname{Riv}_{\mathrm{f}}$

$\begin{array}{rr}-0.145 & 0.082 \\ 0.005 & 0.081 \\ -0.023 & 0.086 \\ \mathbf{0 . 1 9 4} & 0.089\end{array}$

$\begin{array}{ll}0.129 & 0.084\end{array}$

$\mathbf{0 . 2 5 6} 0.071$

$\mathbf{- 0 . 2 0 2} 0.082$

$-0.058 \quad 0.085$

$\begin{array}{ll}-0.062 & 0.053\end{array}$

$\begin{array}{ll}-0.049 & 0.056\end{array}$

$-0.007 \quad 0.055$

0.162 0.053

$\begin{array}{ll}\mathbf{0 . 1 6 7} & 0.082\end{array}$

$\mathbf{0 . 2 4 6} 0.074$

$\begin{array}{ll}-0.049 & 0.090\end{array}$

$\begin{array}{ll}-0.027 & 0.095\end{array}$

.095

$<.001$

.015

.220

.035
.450
.415
.015

$-0.310$

$-0.143$

$-0.193$

0.016

0.025

0.170

0.128
0.339

0.128
0.339

$-0.074$

0.262

0.122

$-0.348$

$-0.229$

0.395

$-0.023$

0.100

8 


\begin{tabular}{lrrrrr}
$\operatorname{Adm}_{m}$ & -0.063 & 0.061 & .160 & -0.173 & 0.059 \\
$\operatorname{Adm}_{f}$ & 0.050 & 0.063 & .190 & -0.093 & 0.159 \\
$\operatorname{Riv}_{m}$ & -0.108 & 0.072 & .040 & -0.268 & 0.010 \\
$\operatorname{Riv}_{f}$ & $\mathbf{- 0 . 1 3 2}$ & 0.067 & .015 & -0.263 & -0.015 \\
\hline
\end{tabular}

Note. Cells present unstandardized estimates from multilevel models for dyadic diary data for distinguishable dyads including constrained actor- and partner-effects across gender. Significant effects are highlighted in bold. ${ }^{5}$ Effects of predictors referring to the same gender as the outcome represent actor-effects. Effects of predictors referring to a different gender as the outcome represent partner-effects. Rel.Sat = relationship satisfaction, Adm $=$ narcissistic admiration, $\mathrm{Riv}=$ narcissistic rivalry, $\mathrm{m}=$ male, $\mathrm{f}=$ female, $\mathrm{LCL}=$ lower confidence limit $(95 \%$ CI), UCL $=$ upper confidence limit $(95 \%$ CI $) . N_{\text {within }}=1941, N_{\text {between }}=171$.

\section{Table 4}

Indirect Effects Based on Multiple Mediator Model Linking Narcissistic Admiration and Rivalry to Relationship Satisfaction Through Situation Perception

\begin{tabular}{lrrrrr}
\hline & Estimate & $\begin{array}{c}\text { Posterior } \\
\text { SD }\end{array}$ & $\begin{array}{c}\text { One- } \\
\text { tailed } \\
\text { p-Value }\end{array}$ & LCL & UCL \\
\hline Indirect effects based & on actor-effects & & & \\
Admiration & & & & & \\
Duty & 0.000 & 0.002 & .450 & -0.004 & 0.004 \\
Intellect & $\mathbf{0 . 0 0 7}$ & 0.003 & $<.001$ & 0.003 & 0.014 \\
Adversity & 0.006 & 0.010 & .155 & -0.008 & 0.031 \\
RoMance & $\mathbf{0 . 0 2 4}$ & 0.007 & $<.001$ & 0.010 & 0.039 \\
POsitivity & $\mathbf{0 . 0 5 3}$ & 0.020 & $<.001$ & 0.018 & 0.090 \\
Negativity & 0.000 & 0.008 & .435 & -0.013 & 0.016 \\
Deception & 0.000 & 0.000 & .445 & -0.001 & 0.001 \\
Sociality & 0.004 & 0.005 & .190 & -0.007 & 0.011 \\
Rivalry & & & & & \\
Duty & & & & & \\
Intellect & $\mathbf{0 . 0 0 4}$ & 0.003 & .015 & 0.000 & 0.010 \\
Adversity & -0.002 & 0.003 & .220 & -0.008 & 0.003 \\
RoMance & $\mathbf{- 0 . 0 2 4}$ & 0.015 & .020 & -0.059 & -0.001 \\
POsitivity & -0.002 & 0.009 & .390 & -0.022 & 0.015 \\
Negativity & -0.037 & 0.023 & .040 & -0.085 & 0.006 \\
Deception & -0.002 & 0.016 & .440 & -0.039 & 0.033 \\
Sociality & 0.000 & 0.001 & .340 & -0.003 & 0.002 \\
& $\mathbf{- 0 . 0 0 9}$ & 0.005 & .015 & -0.020 & -0.001
\end{tabular}

\section{Admiration}

\begin{tabular}{|c|c|c|c|c|}
\hline Dutyactor $\rightarrow$ Rel.Sat $_{\text {partner }}$ & 0.000 & 0.001 & .465 & -0.001 \\
\hline Dutypartner $^{\rightarrow}$ Rel.Sat $_{\text {actor }}$ & -0.003 & 0.002 & .035 & -0.008 \\
\hline
\end{tabular}




\begin{tabular}{|c|c|c|c|c|c|}
\hline Intellect $t_{\text {actor }} \rightarrow$ Rel.Sat $_{\text {partner }}$ & 0.001 & 0.002 & .315 & -0.002 & 0.005 \\
\hline Intellect $_{\text {partner }} \rightarrow$ Rel.Sat $_{\text {actor }}$ & 0.004 & 0.003 & .095 & -0.002 & 0.008 \\
\hline Adversityactor $\rightarrow$ Rel.Sat $_{\text {partner }}$ & 0.002 & 0.003 & .165 & -0.003 & 0.008 \\
\hline Adversitypartner $\rightarrow$ Rel.Sat $_{\text {actor }}$ & 0.008 & 0.010 & .150 & -0.009 & 0.03 \\
\hline RoMance $_{\text {actor }} \rightarrow$ Rel.Sat $_{\text {partner }}$ & 0.004 & 0.002 & .010 & 0.001 & 0.00 \\
\hline RoMance $_{\text {partner }} \rightarrow$ Rel.Sat $_{\text {actor }}$ & 0.015 & 0.008 & .015 & 0.001 & 0.03 \\
\hline POsitivityactor $\rightarrow$ Rel.Sat $_{\text {partner }}$ & 0.000 & 0.002 & .435 & -0.003 & 0.00 \\
\hline POsitivitypartner $\rightarrow$ Rel.Sat $_{\text {actor }}$ & 0.031 & 0.020 & .050 & -0.003 & 0.07 \\
\hline Negativityactor $\rightarrow$ Rel.Sat $_{\text {partner }}$ & 0.000 & 0.001 & .275 & -0.003 & 0.00 \\
\hline${\text { Negativitypartner } \rightarrow \text { Rel.Sat }_{\text {actor }}}$ & 0.001 & 0.010 & .435 & -0.018 & 0.02 \\
\hline Deception $_{\text {actor }} \rightarrow$ Rel.Sat $_{\text {partner }}$ & 0.001 & 0.001 & .215 & -0.002 & 0.00 \\
\hline Deception $_{\text {partner }} \rightarrow$ Rel.Satactor & 0.000 & 0.000 & .425 & -0.001 & 0.00 \\
\hline Socialityactor $\rightarrow$ Rel.Sat $_{\text {partner }}$ & 0.001 & 0.001 & .190 & -0.002 & 0.00 \\
\hline Sociality $_{\text {partner }} \rightarrow$ Rel.Sat $_{\text {actor }}$ & -0.005 & 0.004 & .160 & -0.013 & 0.00 \\
\hline
\end{tabular}

Rivalry

\begin{tabular}{|c|c|c|c|c|c|}
\hline Duty $_{\text {actor }} \rightarrow$ Rel.Sat $_{\text {partner }}$ & 0.001 & 0.001 & .190 & -0.001 & 0.004 \\
\hline Dutypartner $\rightarrow$ Rel.Sat $_{\text {actor }}$ & -0.001 & 0.002 & .415 & -0.005 & 0.003 \\
\hline Intellect $_{\text {actor }} \rightarrow$ Rel.Sat $_{\text {partner }}$ & 0.000 & 0.001 & .355 & -0.002 & 0.001 \\
\hline Intellect $_{\text {partner }} \rightarrow$ Rel.Sat $_{\text {actor }}$ & -0.006 & 0.003 & .015 & -0.011 & -0.001 \\
\hline Adversity $_{\text {actor }} \rightarrow$ Rel.Sat $_{\text {partner }}$ & -0.008 & 0.003 & $<.001$ & -0.015 & -0.002 \\
\hline Adversity $_{\text {partner }} \rightarrow$ Rel.Sat $_{\text {actor }}$ & 0.000 & 0.010 & .495 & -0.023 & 0.016 \\
\hline RoMance $_{\text {actor }} \rightarrow$ Rel.Sat $_{\text {partner }}$ & 0.000 & 0.002 & .390 & -0.004 & 0.003 \\
\hline RoMance $_{\text {partner }} \rightarrow$ Rel.Sat $_{\text {actor }}$ & -0.005 & 0.009 & .275 & -0.025 & 0.011 \\
\hline POsitivityactor $\rightarrow$ Rel.Sat $_{\text {partner }}$ & 0.000 & 0.001 & .435 & -0.003 & 0.002 \\
\hline POsitivitypartner $^{\rightarrow} \rightarrow$ Rel.Sat $_{\text {actor }}$ & -0.041 & 0.021 & .025 & -0.093 & 0.000 \\
\hline Negativity $_{\text {actor }} \rightarrow$ Rel.Sat $_{\text {partner }}$ & 0.002 & 0.002 & .250 & -0.003 & 0.006 \\
\hline Negativity $_{\text {partner }} \rightarrow$ Rel.Sat $_{\text {actor }}$ & 0.000 & 0.006 & .440 & -0.013 & 0.014 \\
\hline Deception $_{\text {actor }} \rightarrow$ Rel.Sat $_{\text {partner }}$ & 0.003 & 0.002 & $<.001$ & 0.001 & 0.008 \\
\hline Deception $_{\text {partner }} \rightarrow$ Rel.Sat $_{\text {actor }}$ & 0.000 & 0.001 & .485 & -0.002 & 0.001 \\
\hline Socialityactor $\rightarrow$ Rel.Sat $_{\text {partner }}$ & -0.002 & 0.002 & .015 & -0.007 & 0.000 \\
\hline Socialitypartner $\rightarrow$ Rel.Sat $_{\text {actor }}$ & -0.008 & 0.005 & .040 & -0.020 & 0.001 \\
\hline
\end{tabular}

Note. Cells present unstandardized estimates from multilevel mediation models for dyadic diary data for

distinguishable dyads and constrained actor- and partner-effects. Significant effects are highlighted in bold. ${ }^{5}$

actor $=$ actor-effect, partner $=$ partner-effect, Rel.Sat $=$ relationship satisfaction, $\mathrm{LCL}=$ lower confidence limit

$(95 \% \mathrm{CI}), \mathrm{UCL}=$ upper confidence limit $(95 \% \mathrm{CI}) . N_{\text {within }}=1941, N_{\text {between }}=171$.

All significant indirect effects (ind) based on actor-effects are displayed in Figure 1.

Narcissistic admiration was positively linked with relationship satisfaction through perceiving daily situations as containing more romance, sexuality or love (ind $=0.024, p<.001$, $95 \% \mathrm{CI}[0.010,0.039]$ ), positive, pleasant, nice things (ind $=0.053, p<.001,95 \% \mathrm{CI}[0.018$, 0.090]), and intellectual, aesthetic, profound things (ind $=0.007, p<.001,95 \% \mathrm{CI}[0.003$, 0.014]). Thus, spouses who scored higher on admiration perceived more romance, positivity, 
and intellect in situations and these perceptions related to being more satisfied with the relationship. Narcissistic rivalry was negatively linked with relationship satisfaction through perceiving daily situations as containing more threat, criticism, and accusation (ind $=-0.024$, $p=.020,95 \% \mathrm{CI}[-0.059,-0.001])$ and less sociality (ind $=-0.009, p=.015,95 \% \mathrm{CI}[-0.020,-$ 0.001]). There was one positive effect linking narcissistic rivalry and relationship satisfaction through the perception of duty and work (ind $=0.004, p=.015,95 \% \mathrm{CI}[0.000,0.010]){ }^{4}$ However, because of the multiple negative indirect effects (see Table 4), the total effect between rivalry and relationship satisfaction was, in sum, negative.

Furthermore, results from unconstrained models showed that the positive indirect effects linking narcissistic admiration and relationship satisfaction via perceptions of romance and intellect were evident in female spouses in particular, whereas the negative indirect effect linking narcissistic rivalry and relationship satisfaction through perceiving more threat was pronounced in males (see Table S1g of the Supplement for more detail). In addition, the perceptions of daily situations as containing deceit, lie or dishonesty and as being less positive were significant mediators between male spouses' rivalry and their lower relationship satisfaction (ind $=-0.008, p<.001,95 \% \mathrm{CI}[-0.018,-0.003]$ for Deception, and ind $=-0.083$, $p=.010,95 \% \mathrm{CI}[-0.155,-0.017]$ for pOsitivity).

Although we did not find a significant total effect between narcissism of one partner and relationship satisfaction of the other (partner-effect), there were still a few indirect effects linking a persons' narcissism with the relationship satisfaction of the partner (Table 4). For example, higher rivalry in one partner was associated with perceiving more threat, criticism, and accusation, which in turn was related to lower relationship satisfaction of the other (ind=$0.008, p<.001,95 \% \mathrm{CI}[-0.015,-0.002])$. Results from unconstrained models further revealed that this effect was pronounced in male partners high in rivalry being linked with lower relationship satisfaction in the female partner through his perception of threat (ind $=-0.009$, $p=.005,95 \% \mathrm{CI}[-0.021,-0.001]$, see Supplement S1 for more details). Furthermore, partners 
of spouses high in admiration also experienced more romance which was related to reporting

higher relationship satisfaction (ind $=0.015, p=.015,95 \% \mathrm{CI}[0.001,0.031]$ ).

\section{Figure 1}

Narcissistic Admiration and Rivalry are Differentially Linked With Relationship Satisfaction

Through Specific Dimensions of Situation Perception

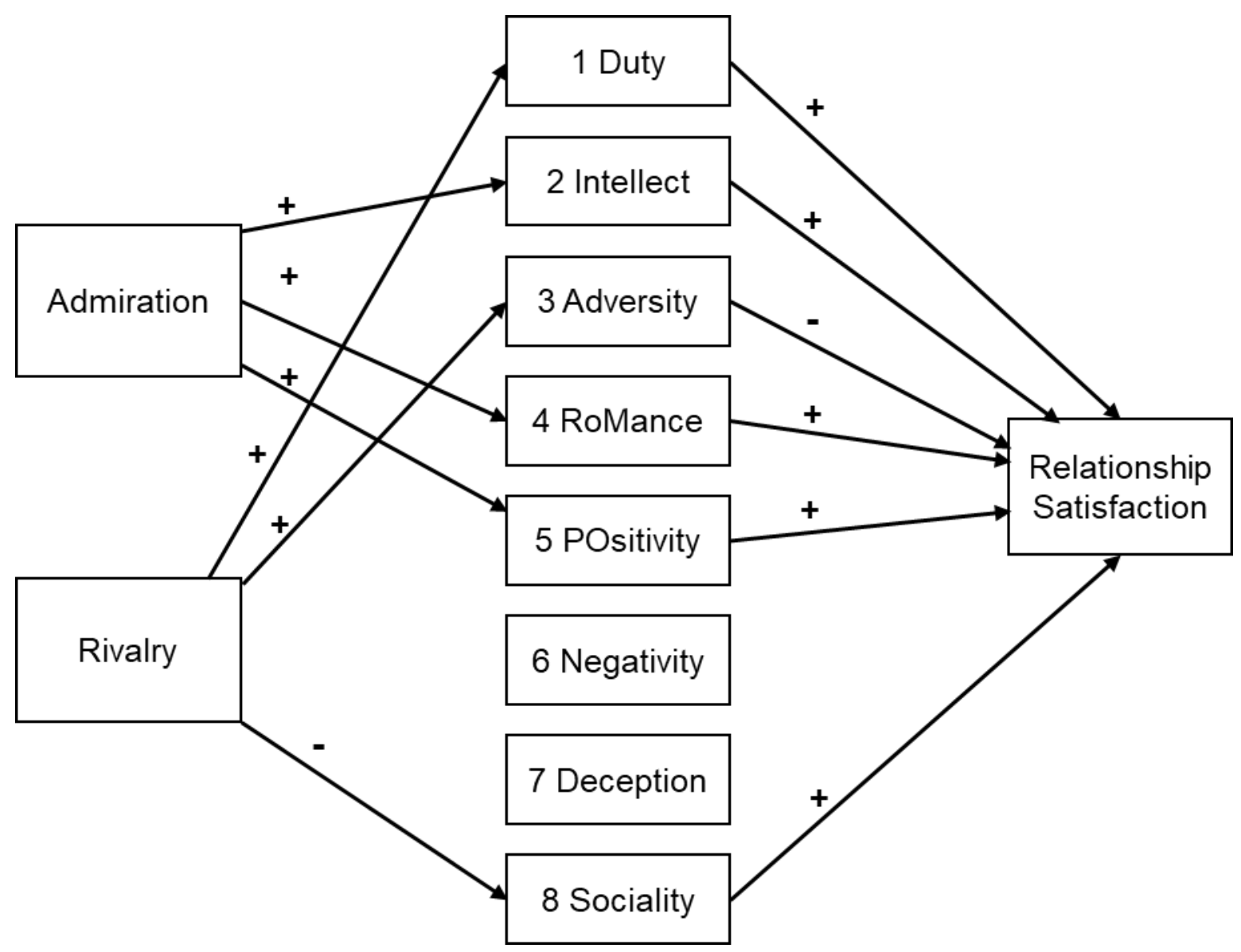

Note. Only paths from significant indirect effects pertaining to actor-effects are displayed.

\section{Discussion}

Using a dyadic diary approach in romantic couples, we replicate previous results (Back et al., 2013) showing that narcissistic admiration is positively and that narcissistic rivalry is negatively related to daily relationship satisfaction. To our knowledge, we are the 
first to demonstrate that these effects are differentially linked through a distinct set of situation perceptions.

In line with our hypotheses, narcissistic admiration was positively linked to relationship satisfaction through perceiving daily situations as containing more positivity (Hypothesis 1) and romance (Hypothesis 5) but not through perceiving more negativity (Hypothesis 2). As expected, we found rivalry to be linked to relationship satisfaction through more perceived adversity (Hypothesis 6) but not through perceiving more negativity (Hypothesis 4). Results did not support a significant association between narcissistic rivalry and relationship satisfaction through less positivity (Hypothesis 5) and perceptions of deception (Hypothesis 7). However, exploratory analyses revealed that these indirect links emerged for male spouses only.

The present study showed that participants higher on admiration and male participants higher on rivalry perceived daily situations as more and less positively connoted, which was associated with higher and lower relationship satisfaction, respectively. The mediation through negativity, however, was not significant for both narcissism facets, probably due to shared variance with positivity. Moreover, supplemental analyses showed that admiration was linked with relationship satisfaction through a "positivity" component (covering variance in the DIAMONDS reflecting positive situation perception) and rivalry was negatively linked with relationship satisfaction through a "negativity" component. Yet, it is noteworthy that that most of the DIAMONDs revealed unique effects on the link between narcissism and relationship satisfaction, even when controlling for a "positivity" and "negativity" component. Consequently, these mediational links can be interpreted as independent of situational valence.

As expected, admiration was uniquely associated with higher relationship satisfaction through perceiving more romantic or sexual content in a situation shared with the partner. Our finding is in line with previous research showing that participants high on agentic narcissism 
(measured with the NPI) perceive situations in a way that allows for flirting and sexual approaches (Sherman et al., 2013). We additionally found that narcissistic admiration was positively related to perceiving situations as intellectually stimulating which was, in turn, positively related to relationship satisfaction. This finding is in line with research demonstrating an association between agentic (NPI) narcissism and intellect-related situational construal (Sherman et al., 2013) and may also hint at a motive inherent in narcissistic admiration to gain social admiration via agentic self-enhancement (Back et al., 2013).

As predicted, rivalry was associated with reduced relationship satisfaction through perceiving more threat, criticism, and accusation. This finding is consistent with previous research showing that people high in rivalry tend to readily perceive ego-threat and others as more aggressive (Back et al., 2013) and less respectful (Vrabel et al., 2019). In addition, rivalry was also associated with reduced relationship satisfaction through perceiving less sociality in situations experienced with one's partner. This finding was unanticipated, yet dovetails with rivalry's relations to lower extraversion (Back et al., 2013) and a higher preference for solitude (Fatfouta, 2017). Whether high levels of rivalry of one or both partners in a romantic relationship are actually linked to a tendency of the couple to spend less time with third parties, however, remains an open question. Since the indirect effects were no longer statistically significant after controlling for positive and negative perception components, they may have been at least partly due to situational valence. Another unexpected finding was rivalry's positive link to relationship satisfaction through higher perceptions of duty. Given that this association was not apparent in the zero-order correlations and not predicted from theory, we suggest replicating it before further interpreting it.

Although we did not find evidence for partner-effects regarding the (total) link between narcissism and the other partner's relationship satisfaction, we were able to identify a few indirect effects linking narcissism to a partner's relationship satisfaction. For example, 
higher rivalry in one partner was associated with experiencing more threat, criticism, and accusation, which in turn was related to lower relationship satisfaction in the other partner. Our results imply that narcissism has an effect on a partner's relationship satisfaction - not directly but indirectly through situation perception. Perhaps, multiple indirect effects linking the same dependent and independent variables but pointing in opposite directions, or multiple indirect effects with only a limited number being statistically significant may have cancelled each other out, resulting in a zero total effect between narcissism and the other partner's relationship satisfaction. Following Hayes (2013) or Shrout and Bolger (2002), a significant total effect is no prerequisite for mediation (or vice versa). Still, we found only a few significant indirect effects including partner-effects. This is important to keep in mind in order to prevent overinterpretation of our findings.

The present study highlights the utility of the NARC model (Back et al., 2013) in two ways. First, our results are in line with the model's core assumption that agentic and antagonistic aspects of narcissism differentially relate to social interaction outcomes. Second, our results underscore the relevance of cognitive processes as mediators of the relationship between trait narcissism and social experiences. Using a daily diary method to examine situation perception, the current research extends the NARC model by providing some insight into the cognitive processes contributing to the social consequences of narcissistic admiration and rivalry. Thereby, our findings add to the process-based conceptualization of agentic and antagonistic narcissism and might also help to understand how trait narcissism stabilizes or changes over time (see Back, 2018). For example, in terms of person-situation transactions, frequent perceptions of mistrust or deception may provoke negative reactions from relationship partners which may then further reinforce mistrust and stabilize antagonistic personality (for a similar argument regarding justice sensitivity, see Gollwitzer et al., 2015).

\section{Limitations and Future Research}


While the use of a broad range of daily situation perceptions when focusing on the DIAMONDS (Rauthmann et al., 2014) adds to the study`s ecological validity, future research should investigate different taxonomies of situational experiences such as CAPTION (measuring Complexity, Adversity, Positive valence, Typicality, Importance, Humor, and Negative valence of a situation; Parrigon et al., 2017), Situation Five (measuring outcomeexpectancy, briskness, cognitive load, psychological and physical load, and lack of stimuli in situations; Ziegler et al., 2019), or SIS (the Situational Interdependence Scale, measuring mutual dependence, power, conflict, future interdependence, and information certainty; Gerpott et al., 2018). Since the latter was specifically developed to describe social situations, it might be of pronounced value for the investigation of interpersonal dynamics; for example, the perception of power discrepancies is not considered within the DIAMONDS taxonomy but might be crucial to understand narcissistic situational construal (e.g., Grapsas et al., 2020).

In the present research, we identify distinct situational perceptions as mediators of the association between relationship satisfaction and agentic and antagonistic facets of grandiose narcissism. There are, however, several other attempts to parse the narcissistic personality construct (e.g., Crowe et al., 2019; Krizan \& Herlache, 2018; Rogoza et al., 2019; Rose, 2002; Wright \& Edershile, 2018) and we would like to encourage researchers to investigate communal aspects of grandiose narcissism characterized by overly positive self-views regarding one's own communion (e.g., morality, prosociality, and interpersonal aptitude; Gebauer et al., 2012; Rentzsch \& Gebauer, 2019) or vulnerable forms of narcissism, such as hypersensitive narcissism, pathological narcissism, and narcissistic personality disorder (Miller et al, 2018). Furthermore, our focus was on romantic relationships in Western societies. Future work may want to investigate different forms of close relationships, for example friendships (Maass et al., 2018), and to examine whether the present findings generalize to, for example, collectivistic cultures. 
Importantly, the present data do not allow to differentiate between situational construal and objective features of the situation. As outlined above, it is unclear whether those higher in antagonistic narcissism only perceive the situation as involving lower sociality or whether couples with at least one partner high in antagonistic narcissism are indeed less likely to socialize with others. In supplemental analyses, we made a step towards disentangling subjective situation construal from characteristics of the situations shared by both relationship partners (situation contact; see also Rauthmann, Sherman et al., 2015). We showed that most of the indirect effects linking narcissism to relationship satisfaction were due to both, subjective situation construal and situation contact. Still, future work should investigate whether the objective features of daily situations differ between people high or low in narcissism and what role self-selection and behaviors used to actively shape situations play.

Our findings of indirect effects linking a partner's narcissism to the other spouses' relationship satisfaction (partner-effect) imply that either narcissism or situation perception must at a certain point also translate into observable behavior. For example, perceiving more threat, criticism, and accusation may be followed by observable aggressive behavior which in turn is linked to reduced relationship satisfaction in the partner. Moreover, partners high in admiration may behave very charmingly which may foster experiences of romance in the other partner which in turn relates to higher relationship satisfaction. While beyond the scope of the present study, future research should explicitly test such behavioral-cognitive pathways when it comes to the association between narcissism and romantic partners' relationship outcomes.

Finally, an important limitation is that the present results do not allow conclusions about causality. Future research may want to focus on causal testing. For example, researchers might manipulate situational settings with a moderate vs. strong affordance level in the laboratory (e.g., Blum et al., 2018) and investigate the corresponding perceptions of romantic partners. 


\section{References}

Arslan, R. C., Walther, M. P., \& Tata, C. S. (2019). formr: A study framework allowing for automated feedback generation and complex longitudinal experience sampling studies using R. Behavior Research Methods. Advance Online Publication. https://doi.org/10.3758/s13428-019-01236-y.

Back, M. D. (2018). The Narcissistic Admiration and Rivalry Concept. In A. D. Hermann, A. Brunell, \& J. Foster (Eds.), The handbook of trait narcissism: Key advances, research methods, and controversies (pp. 57-67). Springer.

Back, M. D., Dufner, A. C. P., Gerlach, T. M., Rauthmann, J. F., \& Denissen, J. J. A. (2013). Narcissistic admiration and rivalry: Disentangling the bright and dark sides of narcissism. Journal of Personality and Social Psychology, 105(6), 1013-1037. https://doi.org/10.1037/a0034431

Blum, G. S., Rauthmann, J. F., Göllner, R., Lischetzke, T., \& Schmitt, M. (2018). The Nonlinear Interaction of Person and Situation (NIPS) model: Theory and empirical evidence. European Journal of Personality, 32(3), 286-305. https://doi.org/10.1002/per.2138

Bolger, N., \& Laurenceau, J. P. (2013). Intensive Longitudinal Methods: An introduction to diary and experience sampling research. Guilford Press.

Campbell, W. K., Bonacci, A. M., Shelton, J., Exline, J. J., \& Bushman, B. J. (2004). Psychological entitlement: Interpersonal consequences and validation of a self-report measure. Journal of Personality Assessment, 83(1), 29-45. https://doi.org/10.1207/s15327752jpa8301_04

Campbell, W. K., \& Campbell, S. M. (2009). On the Self-regulatory Dynamics Created by the Peculiar Benefits and Costs of Narcissism: A Contextual Reinforcement Model and Examination of Leadership. Self and Identity, 8(2-3), 214-232. https://doi.org/10.1080/15298860802505129 
Campbell, W. K., \& Foster, C. A. (2002). Narcissism and commitment in romantic relationships: An investment model analysis. Personality and Social Psychology Bulletin, 28(4), 484-495. https://doi.org/10.1177/0146167202287006

Campbell, W. K., \& Foster, J. D. (2007). The narcissistic self: Background, an extended agency model, and ongoing controversies. In C. Sedikides \& S. J. Spencer (Eds.), The self (pp. 115-138). Psychology Press.

Campbell, W. K., Foster, C. A., \& Finkel, E. J. (2002). Does self-love lead to love for others? A story of narcissistic game playing. Journal of Personality and Social Psychology, 83(2), 340-354. https://doi.org/10.1037/0022-3514.83.2.340

Casale, S., Fioravanti, G., Baldi, V., Flett, G. L., \& Hewitt, P. L. (2019). Narcissism, perfectionistic self-presentation, and relationship satisfaction from a dyadic perspective. Self and Identity. https://doi.org/10.1080/15298868.2019.1707272

Crowe, M. L., Lynam, D. R., Campbell, W. K., \& Miller, J. D. (2019). Exploring the structure of narcissism: Toward an integrated solution. Journal of Personality, 87(6), 11511169. https://doi.org/10.1111/jopy.12464

Dufner, M., Rauthmann, J. F., Czarna, A. Z., \& Denissen, J. J. A. (2013). Are narcissists sexy? Zeroing in on the effect of narcissism on short-term mate appeal. Personality and Social Psychology Bulletin, 39(7), 870-882. https://doi.org/10.1177/0146167213483580

Fatfouta, R. (2017). To be alone or not to be alone? Facets of narcissism and preference for solitude. Personality and Individual Differences, 114, 1-4. https://doi.org/10.1016/j.paid.2017.03.047

Finn, C., Mitte, K., \& Neyer, F. J. (2013). The relationship-specific interpretation bias mediates the link between neuroticism and satisfaction in couples. European Journal of Personality, 27(2), 200-212. https://doi.org/10.1002/per.1862 
Furr, R. M., \& Funder, D. C. (in press). Persons, situations, and person-situation interactions. In O. P. John \& R. W. Robins (Eds.), Handbook of personality: Theory and research (4th ed.). Guilford.

Gebauer, J. E., Sedikides, C., Verplanken, B., \& Maio, G. R. (2012). Communal narcissism. Journal of Personality and Social Psychology, 103(5), 854-878. https://doi.org/10.1037/a0029629

Gerpott, F. H., Balliet, D., Columbus, S., Molho, C., \& de Vries, R. E. (2018). How do people think about interdependence? A multidimensional model of subjective outcome interdependence. Journal of Personality and Social Psychology, 115(4), 716-742. https://doi.org/10.1037/pspp0000166

Gewirtz-Meydan, A., \& Finzi-Dottan, R. (2018). Narcissism and relationship satisfaction from a dyadic perspective: The mediating role of psychological aggression. Marriage \& Family Review, 54(3), 296-312. https://doi.org/10.1080/01494929.2017.1359814

Gollwitzer, M., Süssenbach, P., \& Hannuschke, M. (2015). Victimization experiences and the stabilization of victim sensitivity. Frontiers in Psychology, 6. https://doi.org/10.3389/fpsyg.2015.00439

Grapsas, S., Brummelman, E., Back, M. D., \& Denissen, J. J. A. (2020). The “why” and "how" of narcissism: A process model of narcissistic status pursuit. Perspectives on Psychological Science, 15(1), 150-172. https://doi.org/10.1177/1745691619873350

Guillaume, E., Baranski, E., Todd, E., Bastian, B., Bronin, I., Ivanova, C., Cheng, J. T., de Kock, F. S., Denissen, J. J. A., Gallardo, P. D., Halama, P., Han, G. Q., Bae, J., Moon, J., Hong, R. Y., Hřebíčková, M., Graf, S., Izdebski, P., Lundmann, L., ... Funder, D. C. (2016). The world at 7:00: Comparing the experience of situations across 20 countries. Journal of Personality, 84(4), 493-509. https://doi.org/10.1111/jopy.12176

Hayes, A. F. (2013). Introduction to mediation, moderation, and conditional process analysis: A regression-based approach. Guilford Press. 
Hendrick, S. S. (1981). Self-disclosure and marital satisfaction. Journal of Personality and Social Psychology, 40(6), 1150-1159. https://doi.org/10.1037/0022-3514.40.6.1150

Holtzman, N. S., \& Strube, M. J. (2010). Narcissism and attractiveness. Journal of Research in Personality, 44(1), 133-136. https://doi.org/10.1016/j.jrp.2009.10.004

Hong, R. Y., Chan, W. Y., \& Lim, J. Y. R. (2020). Pathological personality traits and the experience of daily situations. Clinical Psychological Science, 8(2), 333-342. https://doi.org/10.1177/2167702619894902

Jauk, E., Neubauer, A. C., Mairunteregger, T., Pemp, S., Sieber, K. P., \& Rauthmann, J. F. (2016). How alluring are dark personalities? The dark triad and attractiveness in speed dating. European Journal of Personality, 30(2), 125-138. https://doi.org/10.1002/per.2040

Kenny, D. A., Kashy, D. A., \& Cook, W. L. (2006). Dyadic data analysis. Guilford Press. Krizan, Z., \& Herlache, A. D. (2018). The Narcissism Spectrum Model: A synthetic view of narcissistic personality. Personality and Social Psychology Review, 22(1), 3-31. https://doi.org/10.1177/1088868316685018

Laurenceau, J.-P., \& Bolger, N. (2012). Analyzing diary and intensive longitudinal data from dyads. In M. R. Mehl \& T. S. Conner (Eds.), Handbook of Research Methods for Studying Daily Life (pp. 407-422). Guilford Press.

Maass, U., Wehner, C., \& Ziegler, M. (2018). Narcissism and friendships. In A. D. Hermann, A. B. Brunell, \& J. D. Foster (Eds.), Handbook of trait narcissism (pp. 345-354). Springer.

Miller, J. D., Lynam, D. R., Vize, C., Crowe, M., Sleep, C., Maples - Keller, J. L., ... \& Campbell, W. K. (2018). Vulnerable narcissism is (mostly) a disorder of neuroticism. Journal of Personality, 86(2), 186-199. https://doi.org/10.1111/jopy.12303 
Morf, C. C., \& Rhodewalt, F. (2001). Unraveling the paradoxes of narcissism: A dynamic self-regulatory processing model. Psychological Inquiry, 12(4), 177196. https://doi.org/10.1207/S15327965PLI1204_1

Muthén, B. (2010). Bayesian analysis In Mplus: A brief introduction. Retrieved from https://www.statmodel.com/download/

Muthén, L. K., \& Muthén, B. (1998-2017). Mplus User’s Guide (8th ed.). Muthén \& Muthén. Parrigon, S., Woo, S. E., Tay, L., \& Wang, T. (2017). CAPTION-ing the situation: A lexically-derived taxonomy of psychological situation characteristics. Journal of Personality and Social Psychology, 112(4), 642-681. https://doi.org/10.1037/pspp0000111

Peterson, J. L., \& DeHart, T. (2014). In defense of self-love: An observational study on narcissists' negative behavior during romantic relationship conflict. Self and Identity, 13(4), 477-490. https://doi.org/10.1080/15298868.2013.868368

Preacher, K. J., Zyphur, M. J., \& Zhang, Z. (2010). A general multilevel SEM framework for assessing multilevel mediation. Psychological Methods, 15(3), 209-233. https://doi.org/10.1037/a0020141

Rauthmann, J. F., Gallardo-Pujol, D., Guillaume, E. M., Todd, E., Nave, C. S., Sherman, R. A., Ziegler, M., Jones, A. B., \& Funder, D. C. (2014). The Situational Eight DIAMONDS: A taxonomy of major dimensions of situation characteristics. Journal of Personality and Social Psychology, 107(4), 677-718. https://doi.org/10.1037/a0037250

Rauthmann, J. F., \& Sherman, R. A. (2016). Ultra-brief measures for the situational eight DIAMONDS domains. European Journal of Psychological Assessment, 32(2), 165174. https://doi.org/10.1027/1015-5759/a000245 
Rauthmann, J. F., Sherman, R. A., \& Funder, D. C. (2015). Principles of situation research: Towards a better understanding of psychological situations. European Journal of Personality, 29(3), 363-381. https://doi.org/10.1002/per.1994

Rauthmann, J. F., Sherman, R. A., Nave, C. S., \& Funder, D. C. (2015). Personality-driven situation experience, contact, and construal: How people's personality traits predict characteristics of their situations in daily life. Journal of Research in Personality, 55, 98-111. https://doi.org/10.1016/j.jrp.2015.02.003

Reis, H. T., \& Holmes, J. G. (2019). Perspectives on the situation. In K. Deaux \& M. Snyder (Eds.), The Oxford Handbook of Personality and Social Psychology (2nd ed., pp. 6795). Oxford University Press.

Rentzsch, K., \& Gebauer, J. E. (2019). On the popularity of agentic and communal narcissists: The tit-for-tat hypothesis. Personality and Social Psychology Bulletin, 45(9), 1365-1377. https://doi.org/10.1177/0146167218824359

Rogoza, R., Cieciuch, J., Strus, W., \& Baran, T. (2019). Seeking a common framework for research on narcissism: An attempt to integrate the different faces of narcissism within the circumplex of personality metatraits. European Journal of Personality, 33(4), 437455. https://doi.org/10.1002/per.2206

Rose, P. (2002). The happy and unhappy faces of narcissism. Personality and Individual Differences, 33(3), 379-391. https://doi.org/10.1016/S0191-8869(01)00162-3

Sander, J., \& Böcker, S. (1993). Die Deutsche Form der Relationship Assessment Scale (RAS): Eine kurze Skala zur Messung der Zufriedenheit in einer Partnerschaft [The German version of the Relationship Assessment Scale (RAS): A short scale for measuring satisfaction in a dyadic relationship]. Diagnostica, 39(1), 55-62.

Sedikides, C., Rudich, E. A., Gregg, A. P., Kumashiro, M., \& Rusbult, C. (2004). Are normal narcissists psychologically healthy? Self-esteem matters. Journal of Personality and Social Psychology, 87(3), 400-416. https://doi.org/10.1037/0022-3514.87.3.400 
Sherman, R. A., Nave, C. S., \& Funder, D. C. (2013). Situational construal is related to personality and gender. Journal of Research in Personality, 47(1), 1-14. https://doi.org/10.1016/j.jrp.2012.10.008

Shrout, P. E., \& Bolger, N. (2002). Mediation in experimental and nonexperimental studies: New procedures and recommendations. Psychological Methods, 7, 422-445. https://doi.org/10.1037/1082-989X.7.4.422

Vrabel, J., Zeigler-Hill, V., Sauls, D., \& McCabe, G. (2019). Narcissism and respect in romantic relationships. Self and Identity. https://doi.org/10.1080/15298868.2019.1649305

Wright, A. G. C., \& Edershile, E. A. (2018). Issues resolved and unresolved in pathological narcissism. Current Opinion in Psychology, 21, 74-79. https://doi.org/10.1016/j.copsyc.2017.10.001

Wurst, S. N., Gerlach, T. M., Dufner, M., Rauthmann, J. F., Grosz, M. P., Küfner, A. C. P., Denissen, J. J. A., \& Back, M. D. (2017). Narcissism and romantic relationships: The differential impact of narcissistic admiration and rivalry. Journal of Personality and Social Psychology, 112(2), 280-306. https://doi.org/10.1037/pspp0000113

Ziegler, M., Horstmann, K. T., \& Ziegler, J. (2019). Personality in situations: Going beyond the OCEAN and introducing the Situation Five. Psychological Assessment, 31(4), 567580. https://doi.org/10.1037/pas0000654 


\section{Footnotes}

${ }^{1}$ We termed the fourth scale of the DIAMONDS roMance instead of Mating, since it describes the item wording of the $S 8-I I$ (Rauthmann \& Sherman, 2016) when being filled out by romantic partners more properly ("romance, sexuality, love").

${ }^{2}$ In the preregistration document (https://osf.io/m36rn/?view_only=bb38d0d5f501486f8f63cfebf6c8e040), we specified to intend to recruit 100 couples. We interpreted the anticipated sample size as minimum. With respect to achieving higher statistical robustness of effects and precision of their estimation, we were content with collecting data from a larger sample than registered.

${ }^{3}$ Five same-sex couples and one couple indicating „diverse“ as the gender of one partner participated in the study.

${ }^{4}$ Effects may be reported as significant although the one-tailed p-value is presented as $p=.025$ or a confidence limit of the corresponding $95 \%$ confidence interval is displayed as 0.000 due to rounding numbers to the third decimal. 